\title{
DETERMINAÇÃO DE PARÂMETROS DE MODELOS REOLÓGICOS PARA O MELAÇO DE CANA-DE-AÇÚCAR E DO LEITE DE COCO
}

\author{
T. B. Simões ${ }^{1}$, A. D. N. Dantas ${ }^{2}$, J. da Silva ${ }^{3}$ L. E. da Silva ${ }^{4}$, F. L. H. da Silva ${ }^{5}$, N. A. Costa \\ ${ }^{1,2}$ Alunos de Iniciação Científica da Universidade Federal da Paraíba \\ ${ }^{3}$ Aluno de mestrado do PPGCTA da Universidade Federal da Paraíba \\ ${ }^{4}$ Aluno de doutorado do PPGEM da Universidade Federal da Paraíba \\ ${ }^{5}$ Professores do Departamento de Engenharia Química da Universidade Federal da Paraíba \\ E-mail para contato: thiago_bs_06@hotmail.com
}

RESUMO - É essencial conhecer os parâmetros reológicos de substâncias de interesse da indústria. $\mathrm{Na}$ ciência de alimentos o estudo da reologia é fundamental para o projeto de equipamentos e para o monitoramento e controle de qualidade durante o processamento, uma vez que estão diretamente relacionados a propriedades como textura e aceitação sensorial. Esse trabalho teve como objetivo a determinação de parâmetros de modelos reológicos para o melaço de cana-de-açúcar e do leite de coco utilizando um reômetro eletrônico. Os valores da tensão de cisalhamento foram obtidos a cada 10 segundos, com uma variação da taxa de deformação de 600 a $900 \mathrm{~s}^{-1}$ para o leite de coco e 1 a $50 \mathrm{~s}^{-1}$ para o melaço de cana. As medidas foram realizadas em duplicata. Foi observado um comportamento não linear para o leite de coco, o que o caracteriza como um fluido não-Newtoniano. Já para o melaço de cana, foi observado um comportamento linear no reograma, característico de um fluido Newtoniano.

\section{INTRODUÇÃO}

Ter conhecimento sobre as propriedades de deformação e escoamento de corpos sólidos e fluidos é de extrema importância para a indústria em geral, podendo auxiliar no cálculo do dimensionamento de bombas, tubulações, agitadores, trocadores de calor e homogeneizadores e facilitar o controle de qualidade do produto e prazo de validade para a indústria alimentícia e de cosméticos.

Macosko (1994) define reologia como a ciência que estuda a deformação e o fluxo da matéria, sendo que na prática restringe-se ao estudo das relações fundamentais entre a força aplicada e a deformação provocada, primeiramente nos líquidos. $\mathrm{Na}$ ciência de alimentos é usada para estudar o comportamento dos fluidos como resposta à aplicação de forças, sendo que a maior parte das medidas reológicas é feita baseada na aplicação de tensões de cisalhamento. As características reológicas de um produto estão diretamente relacionadas às suas propriedades de consistência e textura, que por sua vez, influenciam na aceitabilidade do mesmo. De acordo com Steffe (1996) são várias as razões para se conhecer as propriedades 
reológicas dos alimentos, dentre elas destacam-se: efetuar o controle de qualidade de matérias-primas e produtos finais e conhecer a influência dos componentes da formulação.

Oliveira et al. (2008) relatam que é através da determinação de modelos reológicos, relacionando principalmente a tensão de cisalhamento com a taxa de deformação do fluido, que explica-se o seu comportamento. Dentre os modelos reológicos, o mais simples é o newtoniano, que se caracteriza por apresentar uma relação linear entre a tensão de cisalhamento e a taxa de deformação. Tabilo-Munizaga e Barbosa-Cánovas (2005) mencionam que poucos fluidos comportam-se como newtonianos, necessitando de modelos mais complexos para caracterizá-los.

A cana-de-açúcar é uma matéria-prima bastante abundante e de baixo custo, sendo produzida em grande parte do planeta. Ultimamente tem sido muito utilizada, seja na forma de caldo de cana ou melaço de cana, para a produção de bioetanol nos países tropicais e subtropicais, Chen et al. (1997). Segundo Magalhães (2007) o xarope de cana é o produto obtido da evaporação do caldo de cana clarificado, após a concentração é chamado de xarope bruto, este contém substâncias insolúveis e macromoléculas (dextrana) que são responsáveis pelo aumento da viscosidade do xarope e podem ocasionar em problemas nas etapas posteriores de fabricação do açúcar.

Mororó (2007) relata que os derivados do coco no Brasil são matérias-primas importantes na indústria alimentícia, estando presentes em bolachas, doces, iogurtes, sorvetes. Dentre os produtos originários do coco, no mercado brasileiro os que apresentam maiores demandas são o coco inteiro, a água e a polpa do coco verde, amêndoa de coco maduro, o coco ralado e o leite de coco. Este se constitui basicamente como uma emulsão óleo em água, formado a partir do extrato aquoso do endosperma do coco sólido, sendo muito utilizado como ingrediente na culinária brasileira, Sampaio Neta et al. (2012).

Conhecer as características reológicas de insumos como o melaço de cana-de-açúcar e do leite de coco é contribuir para o conhecimento da estrutura molecular, planejamento de equipamentos que serão utilizados para o processamento de tais matérias-primas, auxilia no controle do processo industrial, além de influenciar no controle de qualidade e aceitação das mesmas. Assim, esse trabalho teve como objetivo a determinação de parâmetros de modelos reológicos para o melaço de cana-de-açúcar e do leite de coco utilizando um reômetro eletrônico.

\section{METODOLOGIA}

As matérias-primas utilizadas foram o leite de coco e o melaço de cana adquiridos em supermercado. As análises do comportamento reológico do leite de coco e do melaço de cana foram realizadas no laboratório de fluidos da Recogás, no Campus I da Universidade Federal da Paraíba. O comportamento reológico foi feito com o auxilio de um reômetro Thermo Haake (modelo VT550) com geometria de cilindros concêntricos (MV/MV1). As medidas 


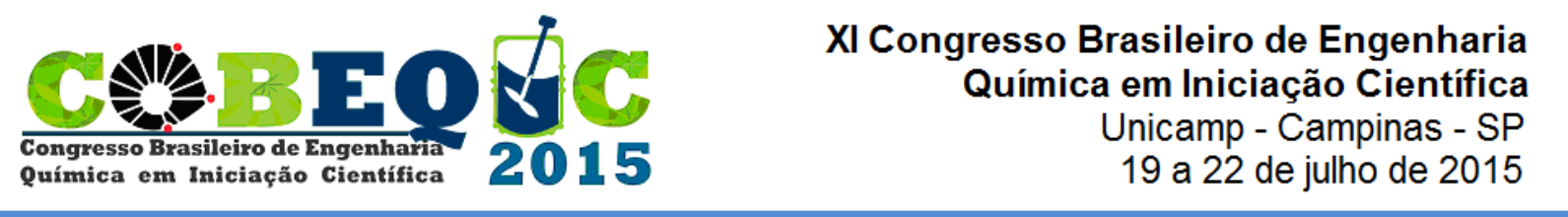

foram realizadas em duplicata a uma temperatura de equilíbrio de $30{ }^{\circ} \mathrm{C}$, por meio de um banho termostático.

Para o controle do processo e registro das medidas efetuadas, utilizou-se o software Rheowin Pro Job Manager. Os valores da tensão de cisalhamento foram obtidos a cada 10 segundos, com uma variação da taxa de deformação de 600 a $900 \mathrm{~s}^{-1}$ para o leite de coco e 1 a $50 \mathrm{~s}^{-1}$ para o melaço de cana. A modelagem reológica foi realizada com o auxílio do software Statistica versão 5.0, ajustando os dados experimentais aos modelos de Newton $(\tau=\mu(\gamma))$, Ostwald-De-Waele $\left(\tau=\mathrm{K}(\gamma)^{\mathrm{n}}\right)$, Bingham $\left(\tau=\tau_{0}+\eta_{\infty}(\gamma)\right)$ e Herschel-Bulkley $\left(\tau=\tau_{0}+\mathrm{K}(\gamma)^{\mathrm{n}}\right)$.

Para verificação da qualidade estatística da regressão dos dados experimentais para determinação dos parâmetros dos modelos reológicos, foram utilizados o coeficiente de determinação $\left(\mathrm{R}^{2}\right)$ e o erro (calculado a partir da raiz quadrada da variância), fornecidos pelo software Statistica versão 5.0.

\section{RESULTADOS E DISCUSSÃO}

A partir da análise dos reogramas do leite de coco (Figura 1) e do melaço de cana (Figura 2), foi observado um comportamento não linear para o leite de coco, o que caracteriza um fluido não-Newtoniano. Já para o melaço de cana, foi observado um comportamento linear no reograma, característico de um fluido Newtoniano.

Figura 1 - Reograma do leite de coco

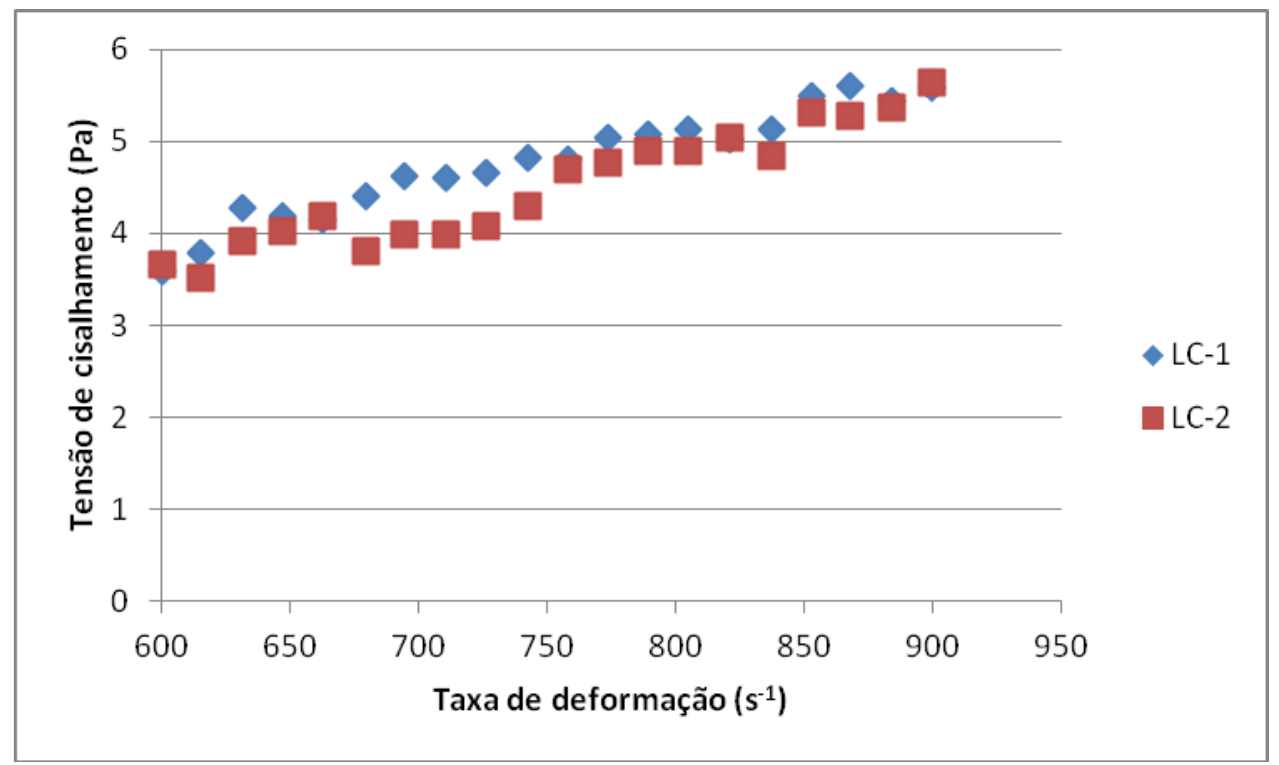




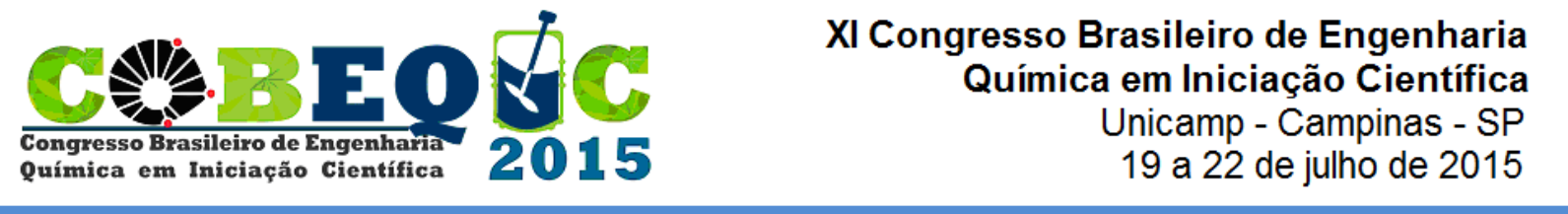

Figura 2 - Reograma do melaço de cana

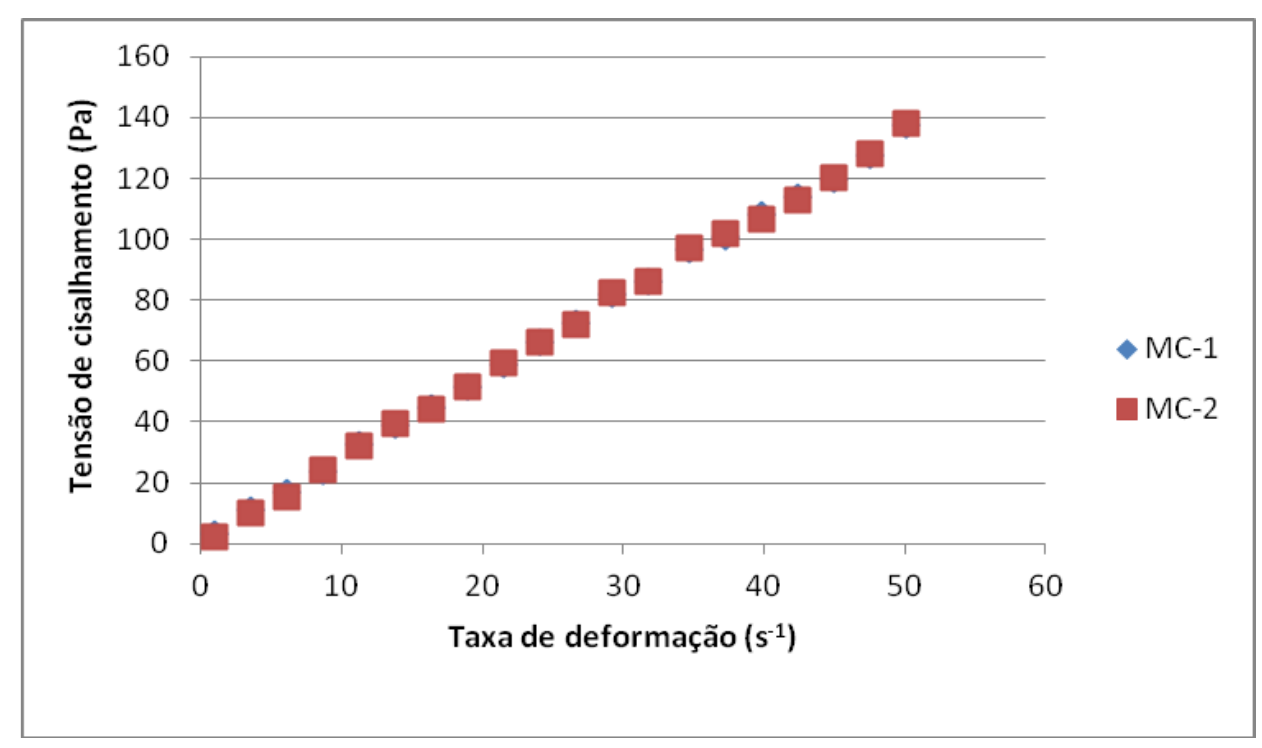

Os parâmetros reológicos, os coeficientes de determinação $\left(\mathrm{R}^{2}\right)$ e os erros estão apresentados na Tabela 1.

Tabela 1 - Parâmetros reológicos das amostras de leite de coco e melaço de cana

\begin{tabular}{|c|c|c|c|c|c|c|c|c|c|}
\hline \multirow{3}{*}{ Amostra } & \multicolumn{9}{|c|}{ Modelo Reológico } \\
\hline & \multicolumn{4}{|c|}{ Newton } & \multicolumn{5}{|c|}{ Ostwald-De-Waele } \\
\hline & \multicolumn{2}{|c|}{$\mu$ (Pa.s) } & \multicolumn{2}{|r|}{ Erro } & K (Pa.s $\left.{ }^{n}\right)$ & $\mathbf{n}$ & $\mathbf{R}^{2}$ & & cro \\
\hline \multirow{4}{*}{$\begin{array}{l}\text { Leite de } \\
\text { Coco } \\
\text { Melaço } \\
\text { de Cana } \\
\end{array}$} & \multicolumn{2}{|l|}{0,006} & 0,942 & 0,206 & \multicolumn{2}{|r|}{1,016} & 0,942 & \multicolumn{2}{|c|}{0,208} \\
\hline & \multicolumn{2}{|l|}{2,720} & 0,999 & 1,393 & 2,917 & 0,980 & 0,999 & \multicolumn{2}{|c|}{1,306} \\
\hline & \multicolumn{4}{|c|}{ Bingham } & \multicolumn{5}{|c|}{ Herschel-Bulkley } \\
\hline & $\tau_{0}(\mathbf{P a})$ & $\begin{array}{c}\eta_{\infty} \\
\left(\text { Pa.s }^{n}\right)\end{array}$ & $\mathbf{R}^{2}$ & Erro & $\tau_{0}(\mathbf{P a})$ & K $\left(\mathbf{P a} . \mathbf{s}^{\mathrm{n}}\right)$ & $\mathbf{n}$ & $\mathbf{R}^{2}$ & Erro \\
\hline $\begin{array}{l}\text { Leite de } \\
\text { Coco }\end{array}$ & $-0,079$ & 0,006 & 0,942 & 0,208 & $-1,147$ & 0,026 & 0,813 & 0,942 & 0,211 \\
\hline $\begin{array}{l}\text { Melaço } \\
\text { de Cana }\end{array}$ & 0,849 & 2,695 & 0,999 & 1,341 & $-0,179$ & 2,952 & 0,977 & 0,999 & 1,322 \\
\hline
\end{tabular}

Confirmando a observação do perfil do reograma do leite de coco que apresenta característica de um fluido não newtoniano, o modelo de Newton não se ajustou adequadamente. Os modelos de Bingham e de Herschel-Bulkley forneceram valores negativos para o $\tau_{0}$, o que não possui nenhum significado físico, tendo em vista que esse parâmetro representa a tensão mínima de cisalhamento que deve ser aplicada para que o fluido comece a escoar. Deste modo, o modelo de Ostwald-De-Waele foi o que mais se 


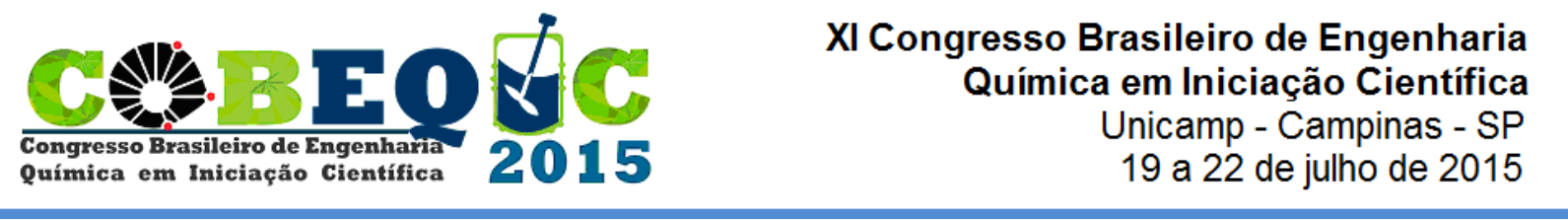

adequou para representar o comportamento reológico do leite de coco. O reograma do melaço mostrou que ele possui um comportamento característico de um fluido Newtoniano.

Observa-se que os dados experimentais em replicatas (Figura 1 e 2) apresentam boa reprodutibilidade.

\section{CONCLUSÃO}

O modelo de Ostwald-De-Waele foi o que mais se adequou para representar o comportamento reológico do leite de coco, enquanto o fluido melaço apresenta um comportamento característico de um fluido Newtoniano. Há excelente reprodutibilidade dos dados experimentais analisados em replicatas.

\section{NOMENCLATURA}

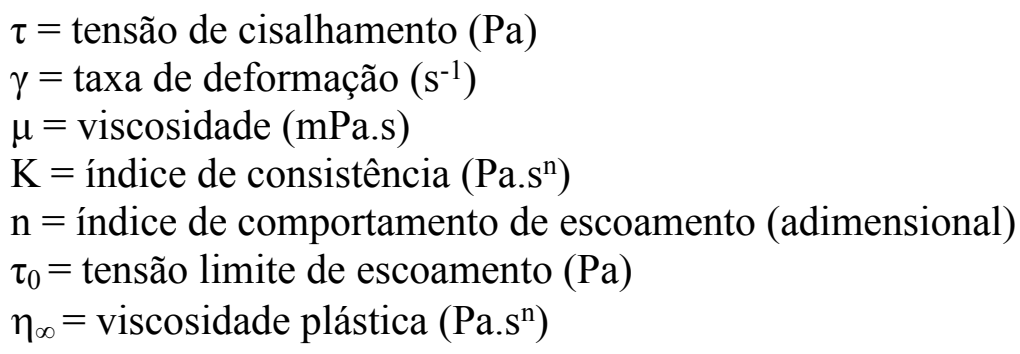

\section{REFERÊNCIAS}

Macosko, C.W. Rheology - Princliples, Measurements and Applications. New York, John Wiley \& Sons Inc. 1994.

Magalhães, A.C.M. Fabricação do Açúcar, 2007. Disponível em: http://www.ebah.com.br/alcool-acucar-unidade-ii-fabricacao-acucar-doc-a-17226.html Acesso em: 25/02/2015.

Mororó, R. Industrialização do coco. Revista tecnol. e trein.. Disponível em: $<$ http://www.tecnologiaetreinamento.com.br>.Acesso em: 25 fevereiro de 2015.

Oliveira, K. H.; de Souza, J. A. R.; Monteiro, A. R. Caracterização reológica de sorvetes. Rheological characterization of ice cream. Ciênc. Tecnol. Aliment., p. 592-598, jul.-set. Campinas, 2008.

Sampaio Neta, N. A.; dos Santos, J. C. S.; Sancho, S. O.; Rodrigues, S.; Gonçalves, L. R. B.; Rodrigues, L. R.; Teixeira, J. A.. Síntese enzimática de ésteres de açúcar e seu potencial como estabilizadores de tensoativos de emulsões de leite de coco. Food Hydrocolloids, p. 324-331, 2012. 
Steffe, J. F. Rheological methods in food process engineering. East Lansing: Fremann Press, 1996.

Tabilo-Munizaga, G.; Barbosa-Cánovas, G. V. Rheology for the food industry. Journal of Food Engineering, v. 67, p. 147-156, 2005. 